\title{
Handedness and space errors
}

\author{
SERGIO CESARE MASIN and ANNA AGOSTINI \\ University of Udine, Udine, Italy
}

\begin{abstract}
Two groups of left- and right-handed subjects compared the lengths of two simultaneous lines presented briefly, one on the left and one on the right of a fixation point. The subjects reported left (right) longer lines in a pair by pressing a key on the left (right) with the left (right) hand. Space errors were almost specular in the two groups. The hand shown to be less preferred according to a handedness test responded more often. Another group of right-handed subjects compared the same lines by responding with crossed arms, so that longer left (right) lines in a pair were now reported with the right (left) hand. Space errors seemed to depend on a motor-pathway preference and perhaps also on an intentional bias.
\end{abstract}

The comparison of two equal stimuli presented successively or simultaneously involves constant errors (Fechner, 1860, 1882). Many explanations of these errors have been proposed, none of which appears to be completely satisfactory (Guilford, 1954; Hellström, 1985; Needham, 1934; Sandusky, 1974; Woodworth \& Schlosberg, 1954). It seems likely that constant errors are difficult to predict, because they depend on multiple factors. We investigated one of these possible factors for simultaneous stimuli.

Usually, constant errors are studied with the method of constant stimuli or of paired comparisons. Let us first review these methods and types of constant errors. In the method of constant stimuli, a standard stimulus (S) is paired with each one of a number of variable stimuli (Vs) varying with respect to a single attribute. Usually, the $S$ is one of the Vs. For each S,V pair, the subject reports which stimulus is greater. The point of subjective equality (PSE) is defined as the value on the continuum of Vs that has $50 \%$ probability of being reported greater than the value of the S. The PSE is thus estimated from the proportions of the "greater" response.

A constant error is defined as the difference between the PSE and the value of the S, and is called time or space error when the $S$ and $V$ in a pair are successive or simultaneous, respectively (Guilford, 1954; Woodworth \& Schlosberg, 1954).

When the method of constant stimuli is applied repeatedly, and the $S$ is set equal to a different $V$ in each repetition, the obtained PSEs vary as an almost linear function of the value of the S, for both successive (Doughty, 1949; Masin, 1987) and simultaneous (Masin, Fanton, \& Crestoni, 1988) stimuli. This function may be called the PSE function. Two PSE functions with different slopes may be distinguished, one for the $S$ preceding and one for the $S$ succeeding the $V$ in a pair. The difference between the slopes of these functions is due to the tenden-

This research was supported by a grant from CNR, the Italian National Research Council. S. C. Masin is now at the University of Padua. Mail may be directed to him at the Department of General Psychology, Piazza Capitaniato 3, 35100 Padova, Italy. cies of PSEs to shift toward the center of the range of $V s$ when the $S$ precedes the $V$ in a pair (Doughty, 1949) and to shift in the opposite direction when the $S$ succeeds the $V$ in the same pair (Fechner, 1860).

The method of paired comparisons allows us to obtain the two PSE functions with a single application. This method involves the use of two identical sets of Vs that vary with respect to a single attribute. Let $A_{i}(i=1,2$, $\ldots, n)$ and $B_{j}(j=1,2, \ldots, n)$ denote these Vs in the two sets, where $i$ and $j$ increase with the varied attribute. For $i=j, A_{i}=B_{j}$. Each $A_{i}$ is paired with each $B_{j}$. For each of the $n^{2}$ ensuing pairs, subjects report whether $A_{i}<B_{j}$ or $A_{i}>B_{j}$. Each $A_{i}$ or $B_{j}$ may be taken as a variable $S$. Therefore, two PSE functions may be distinguished, one when $A_{i}$ is taken as an $S$ (and $B_{1}$ to $B_{n}$ are the Vs), and one when $B_{j}$ is taken as an $S$ (and $A_{1}$ to $A_{n}$ are the Vs). In Figure 1, these functions are shown for $A_{i}$ and $B_{j}$ taken as Ss (open and filled circles, respectively). As is shown in Figure 1a, for pairs of successive lines with $A_{i}$ appearing after $B_{j}$, the slope of the PSE function when $A_{i}$ is taken as an $S$ is greater than the slope of the PSE function when $B_{j}$ is taken as an S (Masin \& Agostini, 1990). And, as is shown in Figure 1b, for pairs of simultaneous lines with $A_{i}$ on the left and $B_{j}$ on the right of a fixation point, the slope of the PSE function when $A_{i}$ is taken as an $S$ is greater than the slope of the PSE function when $B_{j}$ is taken as an $S$ (Masin \& Agostini, 1991). That is, the two PSE functions intersect for both time and space errors.

A factor suggested as a base for time errors is the tendency to favor one verbal comparative response over another (Restle, 1961, pp. 156-159). A verbal response preference has a negligible effect on time errors because a change in mode of judgment leaves the intersection of the PSE functions substantially unaltered (Masin \& Fanton, 1989, Experiment 2). However, it may be that one comparative response becomes favored when there is a preference for one motor pathway, as, for example, when the responses are one verbal and one manual or when both are manual. When both hands are engaged in spatial nonverbal tasks, a shift in hand use seems to occur (Hamp- 


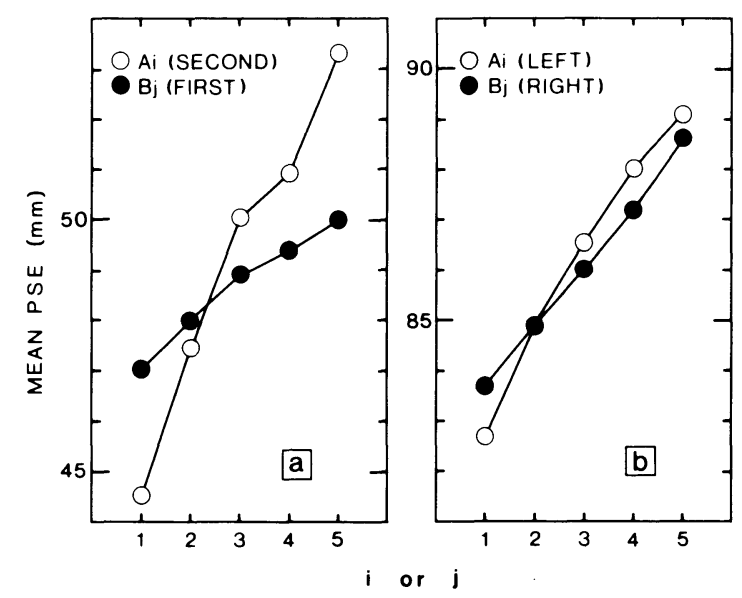

Figure 1. Mean point of subjective equality (PSE) as a function (a) of the standard appearing first $\left(B_{j}\right)$ or second $\left(A_{i}\right)$ in a pair, or (b) appearing on the left $\left(A_{i}\right)$ or right $\left(B_{j}\right)$ of a fixation point (Masin \& Agostini, 1990, 1991).

son \& Kimura, 1984; Wünscher, Jordan, Gille, \& Roth, 1990). Thus, for visual comparisons reported manually, there should be a higher response frequency of the left and right hands in right- and left-handed subjects, respectively. A manual response preference predicts a vertical relative displacement of the PSE functions, in opposite directions for subjects with different hand preferences. In Experiment 1, these predictions were tested with simultaneous stimuli, and with two groups of left- and righthanded subjects.

\section{EXPERIMENT 1}

\section{Method}

Subjects. There were 20 left-handed and 20 right-handed subjects. Handedness was assessed by having subjects report which hand they used for each of 12 common manual activities (Oldfield, 1971). The right-handed subjects were 3 male and 17 female students from the Faculty of Foreign Languages and Literature at the University of Udine. The left-handed subjects were $\mathbf{1 0}$ students from the same faculty and 10 individuals of various professions from outside the university -7 males and 13 females in total. Their declared vision was either normal or corrected to normal.

Stimuli. The stimuli were pairs of simultaneous vertical black lines displayed for $125 \mathrm{msec}$ on a light gray $30 \times 22 \mathrm{~cm}$ IBM 8514 colordisplay screen controlled by an IBM 80 computer. The pairs were combinations of five Vs that were $8.38,8.50,8.62,8.74$, or $8.86 \mathrm{~cm}$ long. The viewing distance was $65 \mathrm{~cm}$. One line in a pair appeared on the left and one on the right of a fixation point, which was a yellow pixel always present in the middle of the screen. Both lines in a pair were $8 \mathrm{~cm}\left(7^{\circ}\right)$ from this pixel. The fixation point and the centers of the two lines lay on an ideal horizontal line. Each pair of lines appeared $2.7 \mathrm{sec}$ after a response was typed and was signaled $1.4 \mathrm{sec}$ before its appearance by a very brief sound.

Illumination level at the screen was $185 \mathrm{~lx}$. So that reflections off the screen could be avoided, the subject viewed the stimuli through a $14.5 \times$ $8.5 \mathrm{~cm}$ rectangular cutout in a black $100 \times 70 \mathrm{~cm}$ cardboard placed $49 \mathrm{~cm}$ from the screen. Eye level was adjusted with a chin- and headrest.

Procedure. The method of paired comparisons was used. Let the Vs on the left and right of the fixation point be $A_{i}$ and $B_{j}$, respectively, with the integers $i$ and $j$ increasing from 1 to 5 with the length of Vs. Each $A_{i}$ was paired with each $B_{j}$. To each subject, the ensuing $25 A_{i}, B_{j}$ pairs were presented randomly, six times consecutively in a single ses- sion, with a 1- to 2-min rest period between the second and third, and the fourth and fifth, presentations of 25 pairs. The subjects did not know that the lines in a pair were to be named $A$ or $B$.

The subjects were asked to fixate the fixation point at the acoustic signal. It was convincingly explained to them that to avoid eye movements during fixation would optimize performance, because it would permit equal visibility of the lines in a pair. The subjects were asked to report manually which line in a pair was longer. They had the left thumb on the leftmost key and the right thumb on the rightmost key of a row of keys on the computer keyboard, which was placed between the subject and the display. The rightmost (leftmost) key had to be pressed with the right (left) thumb when the longer line on the display screen was on the subject's right (left). The "equal" or "don't know" responses were forbidden. Each subject gave 150 responses in total.

\section{Results}

For $i=j, A_{i}=B_{j}$. Consequently, there is one PSE for each $A_{i}$ taken as an S and one PSE for each $B_{j}$ taken as an $S$.

In Figure 2, the mean PSEs are shown as a function of $i$ or $j$, for left $\left(A_{i}\right.$, open circles) and right $\left(B_{j}\right.$, filled circles) Ss, for left-handed (left diagram) and right-handed (right diagram) subjects. These mean PSEs are means of 120 individual PSEs (six replicates) computed by the method of transitions, and correspond substantially to the mean PSEs obtained with the weighted least squares procedure (Masin, 1989). The arrows indicate the Vs. A 2 (preferred hand) $\times 2$ (position of S) $\times 5$ (value of S) analysis of variance (ANOVA) with six replicates showed that the main effects due to preferred hand and position of $S$ were nonsignificant $[F(1,38)=.70$ and .82 , respectively]. The interactions between preferred hand and position of $S[F(1,38)=146]$, preferred hand and value of $S[F(4,152)=20.2]$, position of $S$ and value of $S$ $[F(4,152)=19.3]$, and preferred hand, position of $S$, and value of $S[F(4,152)=8.85]$ were all significant at the 0.0001 level. This ANOVA indicates (1) that, in agreement with Hampson and Kimura's (1984) and Wünscher et al.'s (1990) data, the two PSE functions following an application of the method of paired comparisons are separated and almost specular in left- and right-handed sub-

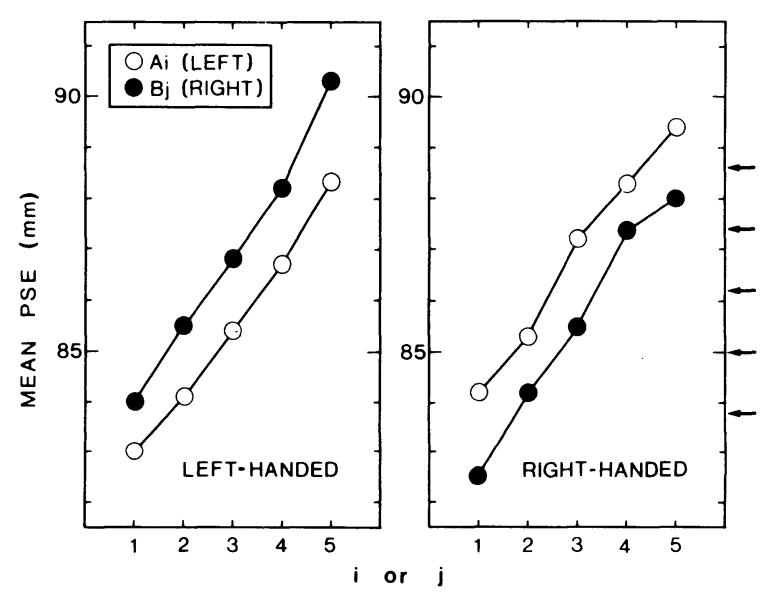

Figure 2. Mean point of subjective equality (PSE) as a function of the standard appearing on the left $\left(A_{i}\right)$ or right $\left(B_{j}\right)$ of a fixation point (Experiment 1: left- and right-handed subjects). 


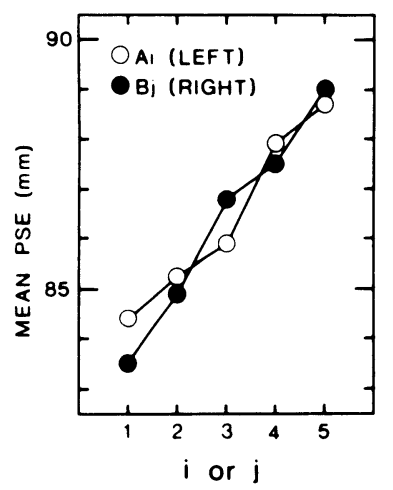

Figure 3. Mean point of subjective equality (PSE) as a function of the standard appearing on the left $\left(A_{l}\right)$ or right $\left(B_{j}\right)$ of a fixation point (Experiment 2: right-handed subjects responding with crossed arms).

jects, and (2) that, contrary to the results in Figure $1 \mathrm{~b}$, the PSE functions for the Ss on the right have a greater slope.

This second result suggests a new factor in space errors. No attempt at explaining this factor will be made here, considering the complex and partly unknown reciprocal influences of the attentional and intentional systems (Verfaellie \& Heilman, 1990).

\section{Discussion}

The results in Figures $1 \mathrm{~b}$ and 2 were obtained from two experiments carried out with the same stimuli, comparative responses, and procedures, but with different motor pathways: verbal and manual, respectively. Thus, it seems likely that the base for space errors is in the response system. Presumably, the results in Figure 2 are due to a motorpathway preference.

Experiment 1 was repeated with right-handed subjects now required to respond with crossed arms. If space errors are caused by a motorpathway preference, the results for the right-handed subjects in Experiments 1 and 2 should differ.

\section{EXPERIMENT 2}

\section{Method}

Subjects. Subjects were 20 new right-handed students -4 males and 16 females recruited as in Experiment 1.

Stimuli. The stimuli were the same as those in Experiment 1.

Procedure. The same keyboard keys as those in Experiment 1 were used for responding. The procedure was the same as that in Experiment 1 , except that now the subjects had to cross their arms so that the right (left) index finger was placed on the leftmost (rightmost) key. During the experiment, half the subjects had the right arm on the left arm, and the other half had the left arm on the right arm. The task was to press the left (right) key with the right (left) index finger when the longer line on the display screen was on the subject's left (right).

\section{Results}

In Figure 3, the means of 120 individual PSEs are shown as a function of $i$ or $j$, for left ( $A_{i}$, open circles) and right $\left(B_{j}\right.$, filled circles) Ss. A 2 (position of $\left.S\right) \times 5$ (value of $S$ ) ANOVA with six replicates showed that the main effects due to position of $S$ were nonsignificant $[F(1,19)=.12]$. The interaction between position of $S$ and value of $S$ was significant $[F(4,76)=53.5$, $p<.0001]$, thus confirming the corresponding interaction in Experiment 1.

\section{GENERAL DISCUSSION}

Since the results in Figures 2 and 3 differ, the interpretation of a motorpathway preference as a source of space errors is corroborated. If this preference is real, some other factor must also be operative. In fact. the relative vertical displacements of the PSE functions in Figure 2 may be interpreted as the effect of two concurrent factors: a motor-pathway preference and an intentional bias toward one visual hemifield. For example, in right-handed subjects, the response frequency of the left hand would be increased due to both a left motor-pathway preference and an intentional bias to respond toward the left visual hemifield. When right-handed subjects cross their arms, the effects of these two factors would balance (Figure 3).

At this stage of research, we can conclude with confidence only that the base for space errors is in the response system. Further research is especially needed to ascertain the role of intention and its possible bias in space errors.

\section{REFERENCES}

Doughty, J. H. (1949). The effect of psychophysical method and context on pitch and loudness functions. Journal of Experimental Psychology, 39, 729-745.

Fechner, G. T. (1860). Elemente der Psychophysik. Leipzig: Breitkopf \& Hartel.

Fechner, G. T. (1882). Revision der Psychophysik. Leipzig: Breitkopf \& Hartel.

GuIlford, J. P. (1954). Psychometric methods. New York: McGraw-Hill.

Hampson, E., KimURA, D. (1984). Hand movement asymmetries during verbal and nonverbal tasks. Canadian Journal of Psychology, 38, 102-125.

Helström, А. (1985). The time-order error and its relatives: Mirrors of cognitive processes in comparing. Psychological Bulletin, 97, 35-61.

MAsin, S. C. (1987). Different biases in the methods of constant and single stimuli. Bulletin of the Psychonomic Society, 25, 379-382.

MAsin, S. C. (1989). A simplified version of the method of transitions. Perceptual and Motor Skills, 68, 131-135.

Masin, S. C., Agostini, A. (1990). Time errors in the method of pair comparisons. American Journal of Psychology, 103, 487-494.

Masin, S. C., AGostini, A. (1991). Attentional scanning and space errors. Manuscript submitted for publication.

MASIN, S. C., FANTON, V. (1989). An explanation for the presentationorder effect in the method of constant stimuli. Perception \& Psychophysics, 46, 483-486.

Masin, S. C., FAnton, V., Crestoni, L. (1988). An experimental study of the asymmetry effect in the method of constant stimuli. Psychological Research, 50, 181-182.

Needham, J. G. (1934). The time-error in comparison judgments. Psychological Bulletin, 31, 229-243.

OLDFIELD, R. C. (1971). The assessment and analysis of handedness: The Edinburgh inventory. Neuropsychologia, 9, 97-113.

Restle, F. (1961). Psychology of judgment and choice: A theoretical essay. New York: Wiley.

SANDUSKY, A. (1974). Memory processes and judgment. In E. C. Carterette \& M. P. Friedman (Eds.), Handbook of perception (Vol. 2, pp. 61-83). New York: Academic Press.

Verfaelle, M., Heilman, K. M. (1990). Hemispheric asymmetries in attentional control: Implications for hand preference in sensorimotor tasks. Brain \& Cognition, 14, 70-80.

WOODWORTh, R. S., \&CHLOSBERG, H. (1954). Experimental psychology. New York: Holt.

Wünscher, T., Jordan, K., Gille, H.-G., Roth, N. (1990). A new approach to cerebral asymmetry: RT differences in simultaneous bimanual finger movement during verbal and nonverbal tasks. Perceptual \& Motor Skills, 70, 543-548.

(Manuscript received December 10, 1990.) 